\title{
Specialized Neuronal and Glial Contributions to Development of the Hamster Lateral Geniculate Complex and Circadian Visual System
}

\author{
G. I. Botchkina and L. P. Morin \\ Department of Psychiatry, Health Science Center, State University of New York, Stony Brook, New York 11794
}

The intergeniculate leaflet (IGL) is an integral part of the adult circadian visual system. It is characterized by the presence of retinal afferents and peptidergic cells projecting via a geniculohypothalamic tract (GHT) to the suprachiasmatic nucleus (SCN), site of the mammalian circadian clock. The adult IGL also contains abundant reactive astrocytes immunoreactive to GFAP. Because glia have a large role in brain development, we examined the ontogeny of the hamster IGL with respect to both glial and neuronal markers. Neuropeptide $Y$-immunoreactive (NPY-IR) cells destined for the IGL appear on embryonic day 11 (E11) in a matrix of vimentin (VIM)-IR radial glia. Migratory ellipsoid NPY-IR cells with long leading and trailing processes become oriented between the reticular thalamic neuroepithelial lobule, and the developing IGL. Most NPY-IR cells arrive in the IGL by E14 and extend axons ventrally into the GHT. These penetrate the SCN at P3 and arborize to an adult-like stage by P10. A specialized GFAP-IR radial glial path coinciding with the migratory route of NPY-IR cells appears by E14. As early as E15, cells contributing to this path are found displaced away from the ventricle. As the glial path disappears from the maturing brain, the entire length of the IGL becomes filled with GFAP-IR astrocytes. These features are consistent with translocation and transformation of a specialized set of radial glia into IGL astrocytes. The results demonstrate that the IGL is a large, developmentally important, feature of the lateral geniculate complex that is embryologically distinct from adjacent dorsal and ventrolateral geniculate nuclei.

[Key words: hamster, intergeniculate leaflet, suprachiasmatic nucleus, reticular thalamic nucleus, ventrolateral geniculate, dorsolateral geniculate, astrocyte, radial glia, neuropeptide $Y$, glial fibrillary acidic protein, vimentin, migration, visual system, development]

The rodent intergeniculate leaflet (IGL) is homologous to the primate pregeniculate nucleus (Moore, 1989) and most of what has been considered ventrolateral geniculate nucleus (VLG) in the cat (R. Y. Moore, unpublished observations). In the hamster, the IGL is a large (about $2 \mathrm{~mm}$ long) component of the lateral geniculate complex. Its rostral terminus lies between the lateral dorsal thalamus, ventrolateral part, and the horizontal cerebral fissure. Caudally, the IGL is contiguous with the lateral terminal

\footnotetext{
Received Jan. 31, 1994; revised May 12, 1994; accepted June 13, 1994.

This work was supported by NIH Grant NS22168 to L.P.M. We thank Ms. J. Blanchard for her continuing technical assistance.

Correspondence should be addressed to Dr. Lawrence P. Morin at the above address.

Copyright $(1995$ Society for Neuroscience $0270-6474 / 95 / 150190-12 \$ 05.00 / 0$
}

nucleus of the accessory optic system (Morin et al., 1992). The IGL receives direct retinal input that is sharply demarcated from the ipsilateral dorsal lateral geniculate (DLG). Immunohistochemistry for serotonin, substance $P$, enkephalin, glutamic acid decarboxylase, and neuropeptide Y (NPY) also reveals a sharp distinction between the DLG and IGL (Morin et al., 1992). Numerous NPY-IR cells identify the IGL and send axons both to the hypothalamic suprachiasmatic nucleus (SCN), site of the mammalian circadian clock (Harrington et al., 1987; Card and Moore, 1989; Morin et al., 1992), and to the posterior limitans thalamic nucleus (Morin and Blanchard, in press), another component of the circadian visual system. Thus, the IGL is a relay nucleus that enables both photic and nonphotic information to modify SCN function and circadian rhythmicity (Johnson et al., 1988; Harrington and Rusak, 1989; Zhang and Rusak, 1989).

The robust, easily measured, locomotor activity of the golden hamster has made this rodent the species of choice for investigating the visual pathways that enable circadian rhythms to by synchronized with the environmental photoperiod (Morin, 1994). These studies have been greatly supported by numerous investigations of hamster visual system development and plasticity (So et al., 1978; Frost et al., 1979; Rhoades et al., 1979; Frost, 1984; Rhoades et al., 1990; Jhaveri et al., 1991). The rodent lateral geniculate complex, composed of DLG, VLG, and the lesser known IGL (Card and Moore, 1989; Morin et al., 1992), is a common anatomical focus of classical and circadian visual system research.

In addition to being intimately connected within the circadian visual system, the SCN and IGL share another common attribute. Both contain dense matrices of persistently reactive astrocytes (Morin et al., 1989). The potential importance of this characteristic has been amplified by the suggestion (Morin et al., 1989) that astrocytes might directly contribute to circadian clock regulation. Recent evidence indicates rhythmic behavior of SCN astrocytes (Lavialle and Servière, 1993). There is also substantial interest in the putative function of neuroglia as constraining agents upon the morphological dynamics of embryonic brain development (Steindler, 1993). In the present article we describe the developmental coordination between transforming neuroglia, lateral migration of NPY-IR neurons to the IGL, and extension of GHT axons to the SCN.

\section{Materials and Methods}

Outbred adult hamsters were acquired (Charles River-Lakeview) and maintained on a $14 \mathrm{hr}$ light $/ 10 \mathrm{hr}$ dark photoperiod (lights off at 2000 $\mathrm{hr}$ ). Timed breeding was performed in the laboratory. The hamster gestation period is $16 \mathrm{~d}$; embryonic day $16(\mathrm{E} 16)=$ postnatal day $0(\mathrm{P} 0)$ $=$ day of birth.

Embryonic and neonatal brains were obtained from anesthetized dams or pups, fixed and cryostat-sectioned at $20 \mu \mathrm{m}$ as previously described 

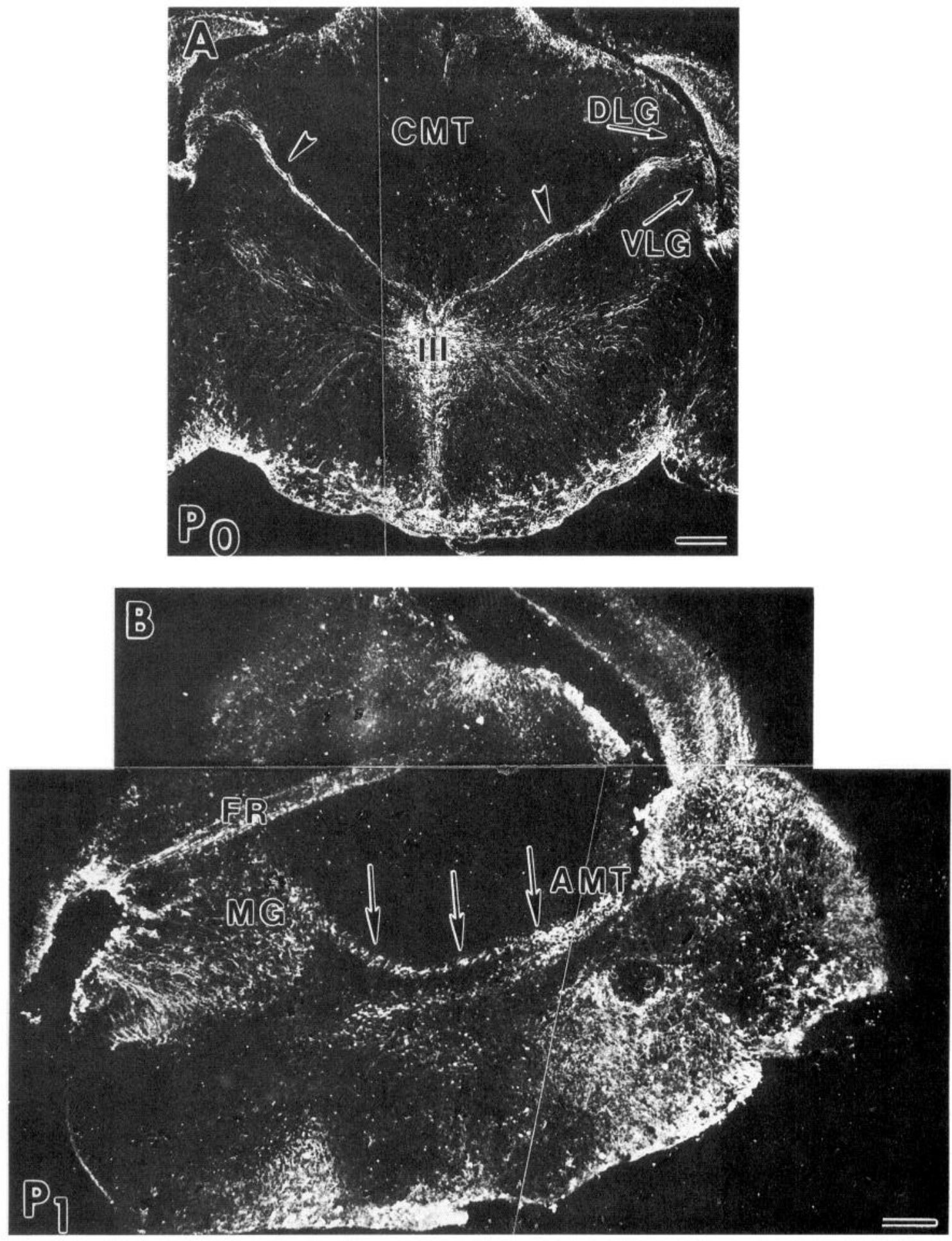

Figure 1. Composite dark-field photomicrographs of the GFAP-IR glial tether. A, Coronal view of the P0 tether (arrowheads) extending between the apex of the third ventricle and the IGL. B, Parasagittal section of the P1 brain showing the large rostrocaudal extent of the tether (arrows). Scale bars, $200 \mu \mathrm{m}$.

(Botchkina and Morin, 1993). Sections were incubated $24 \mathrm{hr}$ at $4^{\circ} \mathrm{C}$ in a dilution (1:2000) of polyclonal rabbit anti-glial fibrillary acidic protein (GFAP; DAKO), anti-NPY (1:2000; Peninsula) or mouse monoclonal anti-vimentin (VIM; 1:200; Accurate) antiserum with $0.3 \%$ Triton X-100 and normal serum after a $2 \mathrm{hr}$ incubation with a blocking solution of $5 \%$ normal serum (goat serum for GFAP and NPY; horse serum for VIM) in 0.05 м PBS. Washed sections were incubated in biotinylated $\mathrm{IgG}$ and avidin-biotin-peroxidase complex (Vector Labs). Reaction product was visualized with $0.01 \%$ hydrogen peroxide and $0.05 \%$ dia- minobenzidine as the chromogen. Specificity of the NPY antiserum has previously been established in adult hamster (Morin et al., 1992), but was tested again using embryonic tissue and preabsorbing the NPY antiserum with $23.6 \mu \mathrm{M}$ NPY. No NPY-IR in the IGL was visible following this procedure. Specificity of VIM and GFAP antisera has been established by their manufacturers.

Tissue to be evaluated using double label immunofluorescence for GFAP/VIM or GFAP/NPY combinations was blocked with normal goat serum and incubated in a mixture of the primary antisera (mouse 

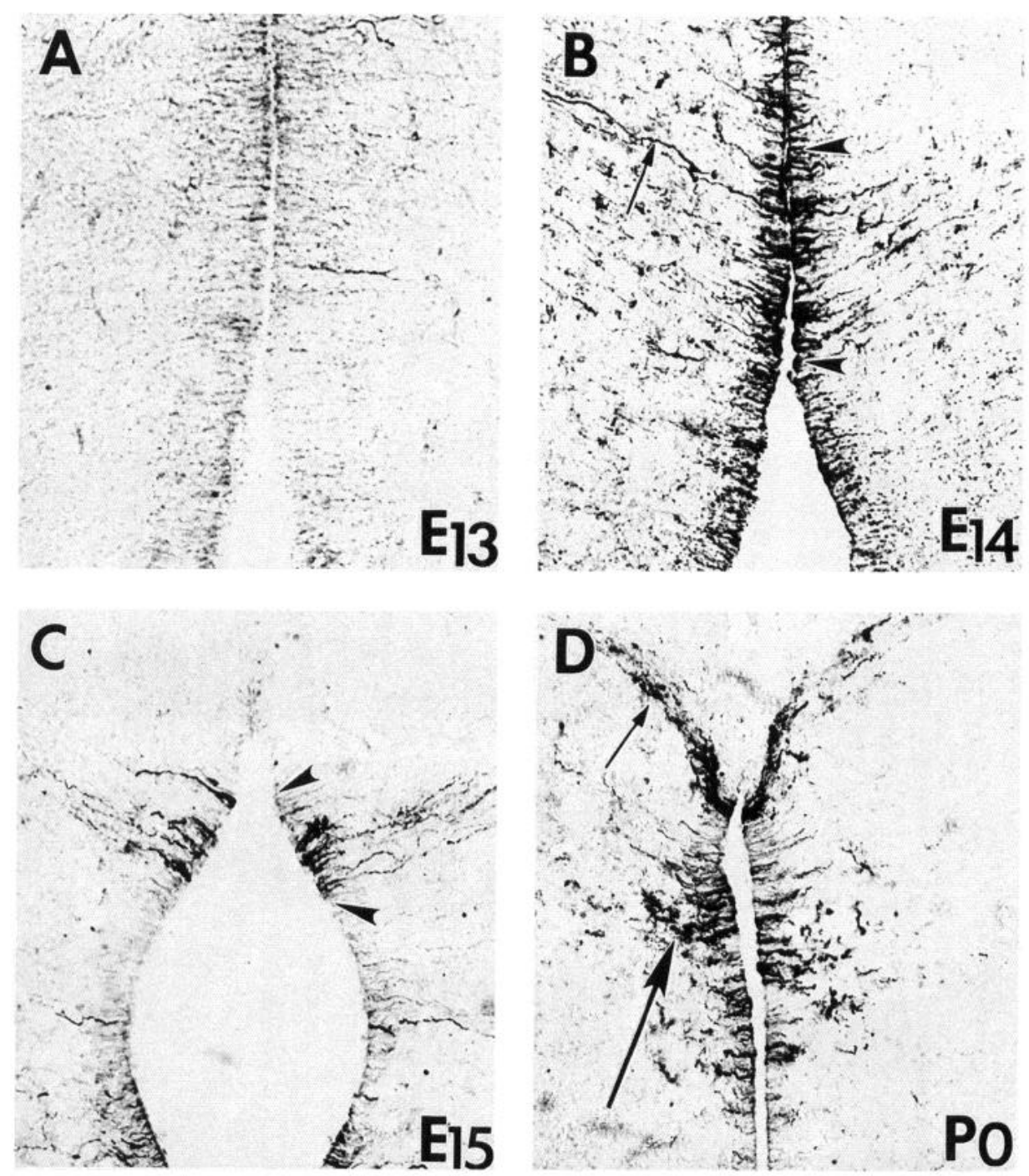

Figure 2. GFAP-IR cells and processes in the ventricular ependyma. $A$, E13. B, E14. Arrowheads demarcate the ventricular germinal zone from which the tether originates and the arrowidentifies a fiber in the tether. $C, \mathrm{E} 15-\mathrm{con}$ solidation of the zone containing the cells of tether origin (arrowheads). D, P0-robust, compact GFAP-IR glial tether (small arrow) and initiation of GFAP-IR in an adjacent set of radial glial cells (large arrow). E, P2-diminution of the tether with loss of reaction product and reduction of number or GFAP-IR cell bodies. $F$, P3-few remaining periventricular GFAP-IR cells and sparse fibers in the tether (arrows).
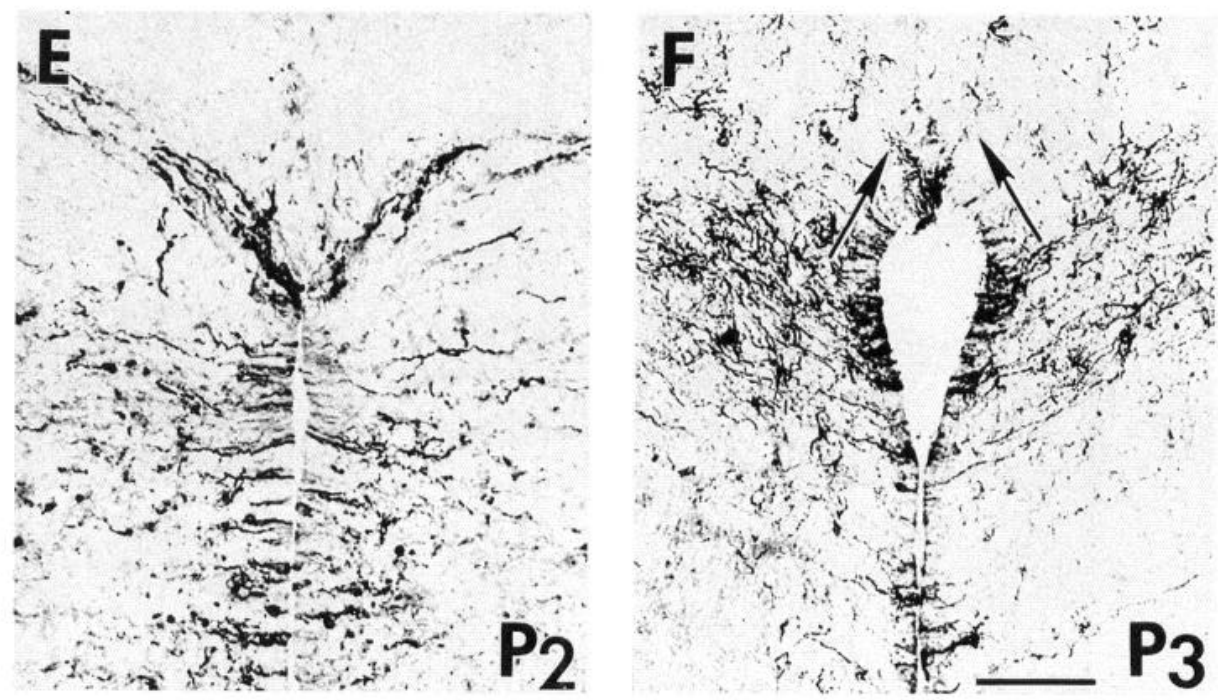

anti-VIM and rabbit anti-GFAP; rabbit anti-NPY and mouse antiGFAP) for $24 \mathrm{hr}$. Sections were rinsed with PBS and incubated in a mixture of the secondary antisera (goat anti-rabbit Texas red and goat anti-mouse FITC). Finally, the tissue was rinsed, air dried, coverslipped with Krystalon, and examined using a Nikon Optiphot equipped with epifluorescence and the appropriate filter cubes.

\section{Results}

GFAP-IR hypothalamic sulcus tether

At E14, long cell processes extending from the RTNL and to the lateral brain surface between the DLG and VLG become 

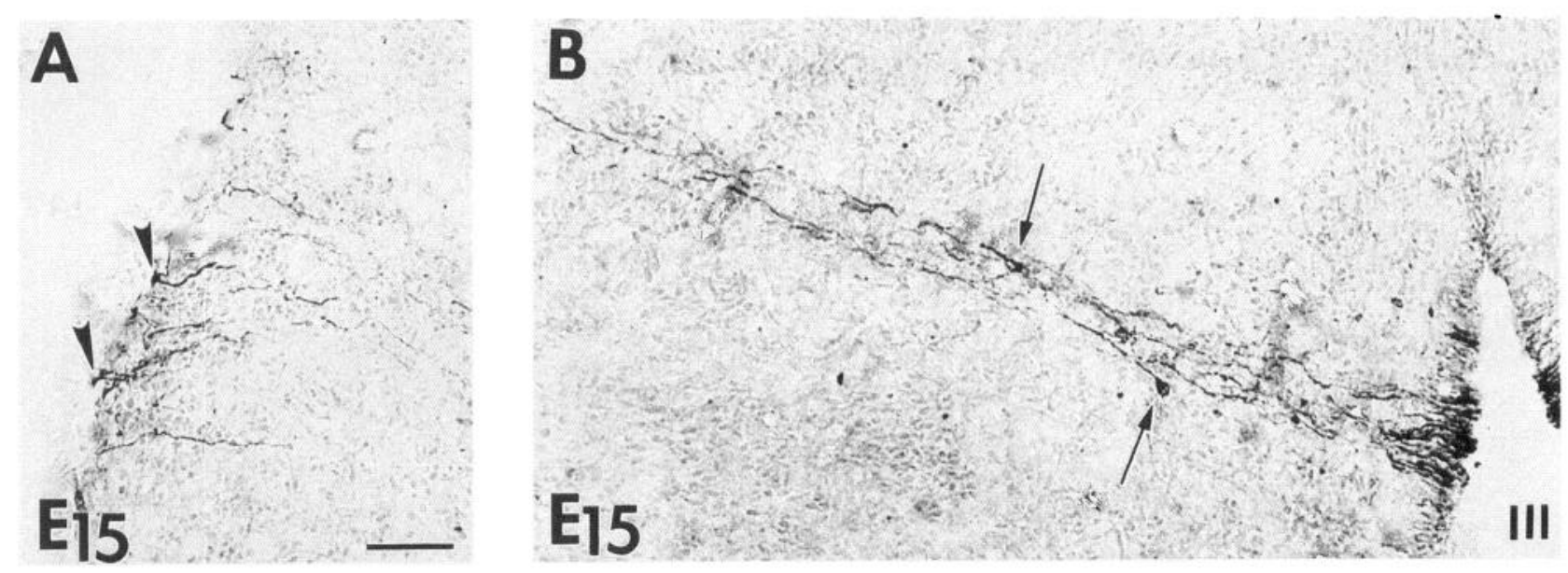

Figure 3. A, GFAP-IR fibers of the tether showing end feet (arrowheads) in brain surface of the lateral geniculate on E15. B, GFAP-IR fibers of the tether and cells (arrows) that are displaced from the ventricular germinal zone on E15. Scale bar, $50 \mu \mathrm{m}$.

visible. The bilateral reticular protuberances merge on E15 to form the roof of the third ventricle. The very distinct, stringlike, bilateral stream of fine GFAP-IR fibers (the "hypothalamic sulcus tether," Silver et al., 1993) is evident in coronal sections (Fig. 1A). Sections made in the sagittal plane brain demonstrate that the tether is actually a sheet, of long rostrocaudal proportions, made of GFAP-IR fibers emanating from a position along the apical third ventricle (Fig. $1 B$ ). Presence of the glial sheet topographically creates dorsal and ventral divisions of the embryonic brain.

No GFAP-IR elements are visible in the developing ventral thalamus before E13 nor are there obvious GFAP-IR cellular elements in the ventricular germinal zone (Fig. 2A). On E14, GFAP-IR appears in cells of the ventricular ependyma near the site of fusion that forms the roof of the third ventricle. The GFAP-IR cells send short processes into the neuropil perpendicular to the ependyma. A few longer fibers are present. The tether becomes very clear on E15, the day of ependymal fusion, with more long processes evident and the ventricular site of origin more narrowly and discreetly defined (Fig. $2 C$ ). Terminal end feet are seen at the lateral brain surface (Fig. $3 A$ ). Beginning on E15, some GFAP-IR cells are clearly displaced from the ventricular germinal zone (Fig. $3 B$ ). At P0-P1, the zone of origin is most narrowly constrained (Fig. $2 D$ ) and the GFAP-IR of the tether attains its most robust appearance (Fig. $1 A$ ). Numerous immunoreactive cells are found within the tether at various distances from the ventricle. Reaction product is substantially reduced in the medial tether by $\mathrm{P} 2-\mathrm{P} 3$ (Fig. $2 E, F$ ) and the entire tether is virtually gone by P5.

\section{VIM-IR radial glia}

The embryonic brain is filled with VIM-IR processes and the lateral geniculate region is no exception (Fig. 4C). VIM-IR radial glia extending through the ventral thalamus remain prominent to P0. At this time, a gradual diminution of visible VIM-IR begins and continues until P21 when no VIM-IR radial glia are found in this region.

Evaluation of adjacent sections suggests that the E15 GFAPIR tether is associated with particular inflections in the course of the VIM-IR radial processes (Fig. $4 A, C$ ) as they approach the lateral geniculate region. The site of tether termination in the lateral geniculate is the IGL, which is identifiable by NPY-
IR neurons (Fig. 4B). Closer study with double immunolabeling of E15 thalamic tissue shows that fibers of the GFAP-IR glial tether are also VIM-IR, but that the vast majority of VIM-IR processes are not GFAP-IR (Fig. $4 D, E$ ).

\section{GFAP-IR astrocytes in the lateral geniculate}

GFAP-IR astrocyte-like cells become evident in the lateral geniculate complex on E15 (Fig. 4A). They are initially found scattered in the dorsal and ventral lateral geniculate areas, slightly medial to the optic tract. A relatively low level of reaction product in cell processes is evident at the brain surface. With age, the density of GFAP-IR in the glia limitans increases and the number of GFAP-IR cells and processes in the DLG or VLG diminishes. At P3, the glia limitans stains darkly and most cell bodies are now located just medial to it. By P5, the glia contributing to the glia limitans have generally vacated the neuropil (Fig. 5A).

In contrast, GFAP-IR astrocyte-like cells become increasingly evident in the terminal region of the tether beginning at P5 (Fig. $5 A$ ). The staining density and morphology of this region gradually assumes the characteristics of the adult IGL (Fig. $5 C, E$ ). In the adult hamster, astrocytes of the lateral geniculate region are concentrated in the IGL (Fig. $6 A$ ). They are found throughout the entire nucleus (Fig. $6 B-G$ ) from its rostral end between the lateral dorsal thalamus, ventrolateral part, and the horizontal cerebral fissure to its caudal end lateral and ventral to the medial geniculate, contiguous with the zona incerta and lateral terminal nucleus.

\section{Origin and migration of NPY-IR neurons}

NPY-IR becomes evident on E11 in small, dot-like neurons without visible processes, which are located between 240 and $460 \mu \mathrm{m}$ lateral to the ventricle (Fig. $7 \mathrm{~A}$ ). At this age and location, the developing diencephalic wall is approximately $500 \mu \mathrm{m}$ thick. On E12, when the diencephalic wall is about $740 \mu$ m thick, the NPY-IR neurons are large and elliptical with long, immunoreactive, leading and trailing processes (Fig. $7 D$ ). Leading processes are seen extending to the lateral thalamic wall. The densest cellular NPY-IR is contained in the axon hillock-like region of the migrating cell. The NPY-IR neurons are found scattered within a dorsolaterally oriented region (Fig. $7 A, B$ ) extending from the reticular thalamic neuroepithelial lobule (RTNL; Alt- 

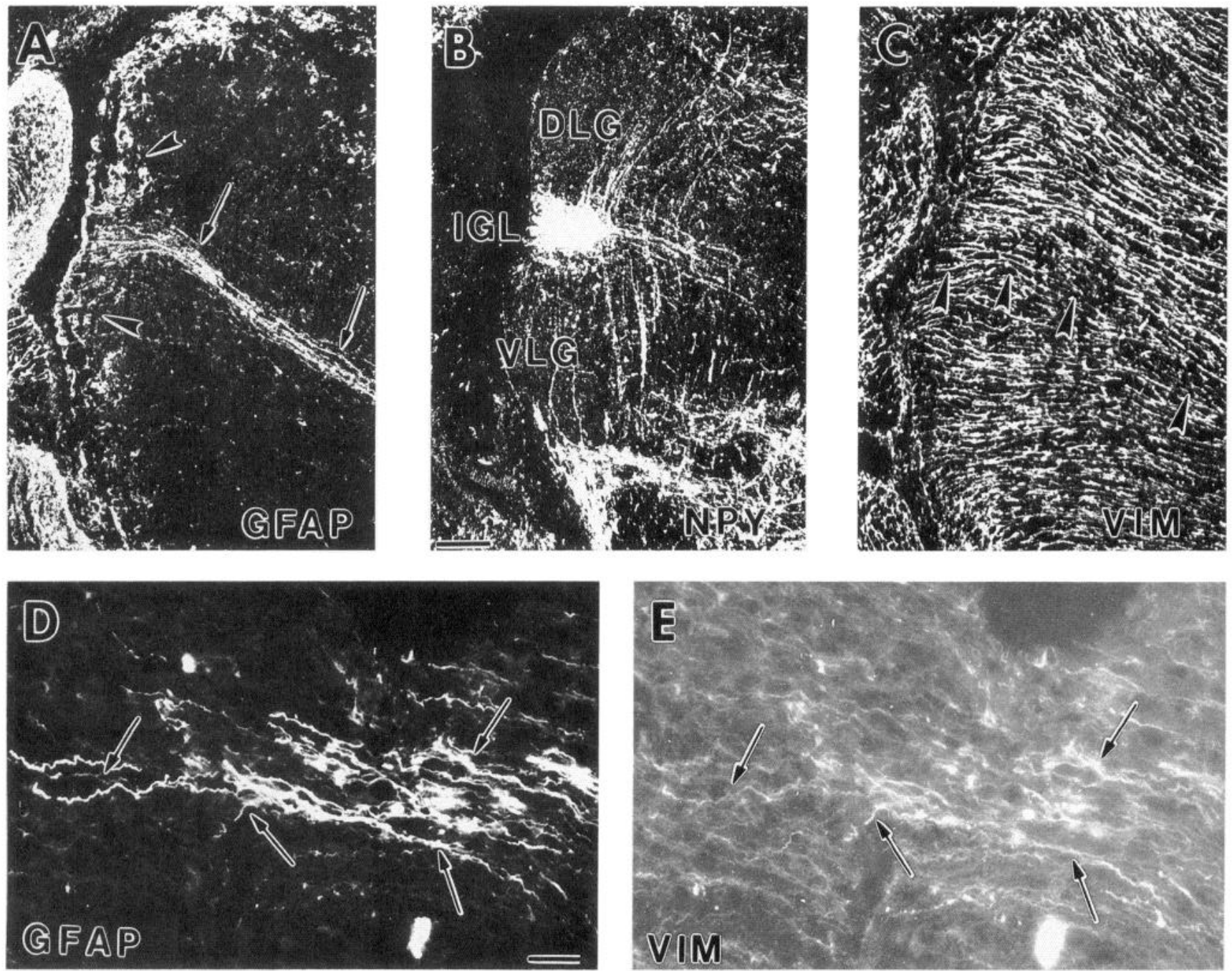

Figure 4. Dark-field comparison of GFAP-IR in the glial tether with location of NPY-IR in the IGL and VIM-IR in the lateral geniculate in adjacent sections of the P0 hamster brain. A, GFAP-IR tether fibers (arrows) entering the lateral geniculate region. Arrowheads point to astrocytelike cells in the developing DLG and VLG. $B$, NPY-IR in the IGL and fibers contributing to the GHT ventrally and fibers extending medial to the DLG dorsally. $C$, VIM-IR radial glia in the lateral geniculate area. Arrowheads delineate the distorted fiber pattern corresponding to the route of GFAP-IR fibers. $D$, Double immunofluorescence showing that GFAP-IR fibers (arrows) in the glial tether are also VIM-IR ( $E$, arrows). Scale bars: $B, 100 \mu \mathrm{m} ; D, 25 \mu \mathrm{m}$.

man and Bayer, 1988). The majority of the cells are found at the medial end of this region, with the major cell axis oriented dorsolaterally. This pattern becomes more extensive on E13, although diencephalic wall thickness remains about $740 \mu \mathrm{m}$. Several cells are found in the lateral geniculate region, adjacent to the optic tract.

By E14, the thickness of the diencephalic wall is about 1000 $\mu \mathrm{m}$ and the locations of NPY-IR cells have changed substantially. Nearly all are grouped adjacent to the optic tract (Fig. $7 \mathrm{~A}, \mathrm{C}$ ) in a position that remains generally constant into adulthood and allows the collection of NPY-IR cells to be identified as constituents of the IGL (Morin et al., 1992). There is a minority of laggard cells that fail to complete the migration to the IGL by E14-P0 (Fig. $8 A$ ). Leading processes of migrated cells are substantially gone; trailing processes are virtually gone by P0. Double label immunohistochemistry (not shown) and examination of adjacent sections (Figs. 4, 5) reacted for GFAPand NPY-IR reveal that the tether passes into the IGL, terminating on the adjacent thalamic surface.
Although NPY-IR cell migration is substantially complete by E14 as GFAP-IR tether visibility is just beginning, a comparison of medial E14 NPY-IR and E15 GFAP-IR can be made (Figs. $3 B, 7 C)$. This shows that the ependymal site of glial origin is just medial to the RTNL and that radial glial fibers giving rise to the tether pass through the site of putative neuronal generation.

\section{GHT formation and innervation of the SCN by NPY-IR processes}

The leading processes of migratory NPY-IR neurons extend to the IGL. As the neurons approach the IGL, the leading processes do not progress in advance of the cell bodies, but apparently become shorter. Many of the NPY-IR cells appear to reorient in a dorsoventral direction upon arrival in the IGL. On E14, reoriented cells with short leading processes can be seen extending both dorsally along the medial DLG (Fig. $4 B$ ) and ventrally (Fig. $8 C$ ). The latter processes are clearly axonal by E15 and extend ventrolaterally contributing to the developing GHT 

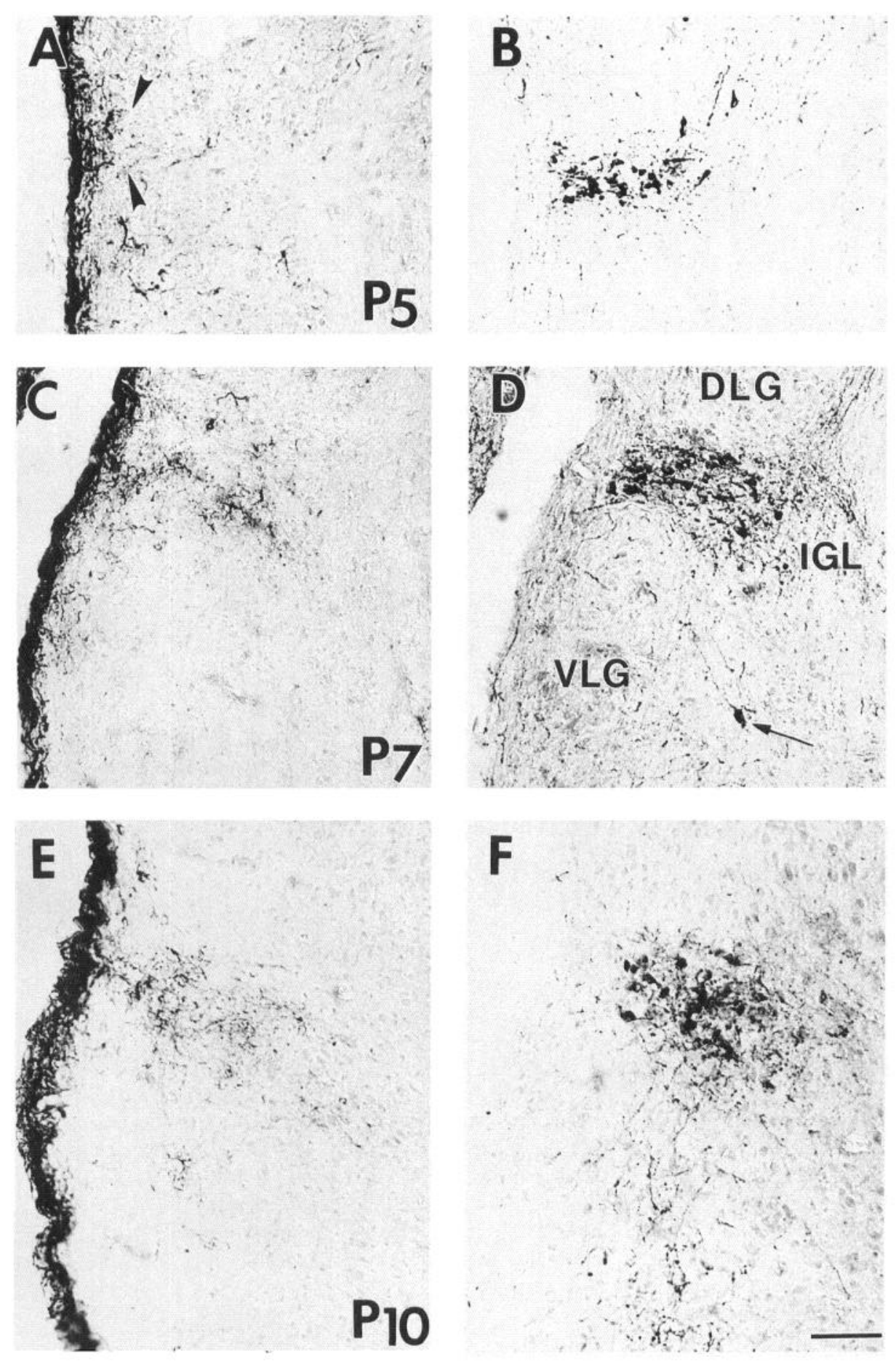

Figure 5. Development of astrocytes $(A, C$, and $E)$ in the IGL on P5, P7, and P10, respectively. Adjacent sections showing NPY-IR cells $(B, D$, and $F$ ) identify the IGL. Arrowheads in $A$ bracket the zone of subsequent astrocyte growth and the arrow in $D$ indicates a NPY-IR cell in the ventral portion of the IGL (Morin et al., 1992). Scale bar, $50 \mu \mathrm{m}$.
(Fig. $8 B$ ). The NPY-IR fibers of the GHT arrive in the ventral SCN on P3 with substantial arborization occurring over the next few days (Fig. $8 D$ ). The geometry of innervation continues to expand and change for 2-3 weeks. However, the NPY-IR innervation of the SCN is substantially similar to the adult pattern by $\mathrm{P} 10$ (Fig. $8 E$ ).

\section{Discussion}

These studies focus attention on several significant issues concerning the general topic of glia-neuron interactions during brain morphogenesis and, more specifically, on development of the lateral geniculate complex and circadian visual system: (1) there is a specific glial structure associated with the development of the lateral geniculate complex; (2) this structure is characterized by the transition from VIM-IR to GFAP-IR; (3) neurons identifiable by specific neuropeptide immunoreactivity associated with a known adult function migrate to their IGL destination in association with the glial structure; (4) the IGL is derived from the same cytogenetic sector as the reticular thalamic nucleus; and (5) extrusion of axons from the IGL peptidergic neurons contribute to a fiber tract in the developing circadian visual system (Fig. 9). 


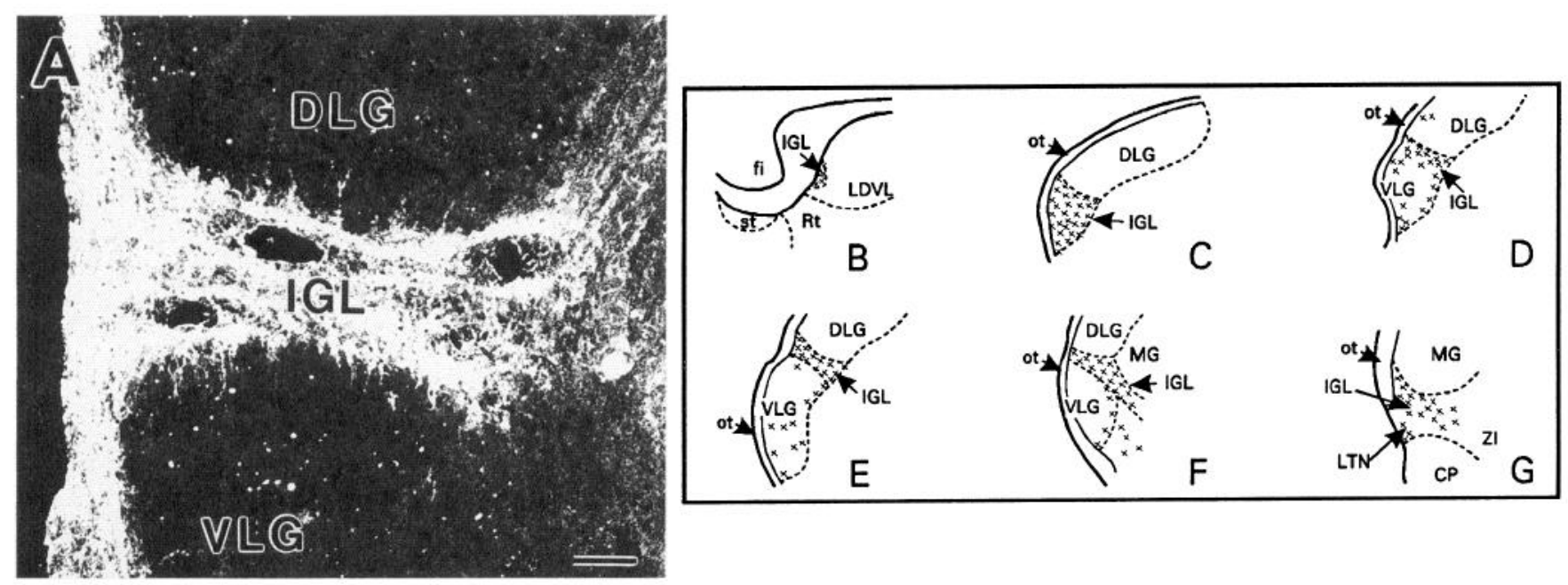

Figure 6. GFAP-IR astrocytes in the adult IGL. $A$, Dark-field coronal photomicrograph showing GFAP-IR astrocytes in the mid-IGL; the blood vessel is always present at this level. Scale bar, $50 \mu \mathrm{m}$. B-F, Schematic distribution of astrocytes (cross-hatching) through the entire hamster IGL.

\section{Radial glia and neuronal migration}

Radial glia provide a biological scaffold for the developing brain (Rakic, 1971, 1972, 1988; Choi and Lapham, 1978; Schmechel and Rakic, 1979; Levitt and Rakic, 1980). Cell bodies of individual radial glia in the ventricular zone contribute single processes terminating with end feet at the outer brain surface (Rakic, 1972; Choi and Lapham, 1978; Schmechel and Rakic, 1979; Voigt, 1989). This scaffold is multidimensional to the extent that glial radiations progressively lengthen as brain dimensions increase. Superimposed is the process of neuronal proliferation and migration (Rakic, 1972). Extensive investigations by Hatten and colleagues (Edmondson and Hatten, 1987; Gregory et al., 1988; Hatten, 1990) have characterized the intimate relationship between a neuron and the individual radial glial process along which it migrates. The present investigations have not directly visualized an equivalent process in the migration of cells to the IGL. However, the long leading and trailing processes and ellipsoid shapes of migrating NPY-IR neurons bear substantial similarity to that which is seen in fixed cortical tissue (Rakic, 1972) or in culture (Edmondson and Hatten, 1987; Gregory et al., 1988). Combined process length of migrating neurons can be hundreds of micrometers (Schmechel and Rakic, 1979).

Neurons that are visible on E11 contain scant NPY-IR and the methods used for these studies are unable to demonstrate processes. We speculate that the NPY-IR neurons are born in the RTNL and initiate migration on E10. The soma becomes identifiable by NPY immunocytochemistry techniques on E11 and the processes identifiable on E12. Thus, the more mature NPY-IR neurons are to be found more laterally in the migratory stream and are the first to reach the IGL. The laggard cells probably contribute to the fairly discrete group of NPY-IR neurons seen in the ventral division of the adult IGL, along the medial border of the VLG (Morin et al., 1992; Morin and Blanchard, 1994).

\section{Astrocyte transformation from radial glia}

Vimentin is the primary structural protein of the radial glial scaffold in rodent brain. Its presence is thought to characterize a particular state of developmental immaturity associated with the migration of neurons (Pixley and de Vellis, 1984). After facilitating neuron migration, VIM-IR radial glia apparently transform into GFAP-IR astrocytes (Choi and Lapham, 1978; Schmechel and Rakic, 1979; Levitt and Rakic, 1980; Voigt, 1989). During a limited transitional period in development or after injury in adult brain, the transforming glia contain both VIM- and GFAP-IR (Pixley and de Vellis, 1984; Voigt, 1989). In the present situation, glial transformation begins on E14 coincident with the arrival of NPY-IR neurons in the IGL. The GFAP-IR tether may represent the specific set of radial glia that, 2-3 d earlier, began to facilitate migration of IGL neurons.

It is not certain whether the glial transformation is a fairly simultaneous action of all cells in the tether or a gradual, cell by cell, state change. It is clear in the present data, however, that the VIM-/GFAP-IR transition occurs only after migration of the NPY-IR cells is virtually complete. Thus, this stage of radial glial transformation toward astrocytic form appears coincident with a specific change in glial function related to neuron migration.

\section{Embryonic origin of IGL cells}

The extensive investigations of thalamic development by Altman and Bayer $(1979 \mathrm{a}-\mathrm{c}, 1988,1989)$ do not recognize an IGL, and there is consequent confusion concerning the development of the lateral geniculate region. At least part of the difficulty is related to the fact that areas previously considered to be medial or rostral divisions of the VLG are actually constituents of the

Figure 7. Origin and migration of NPY-IR cells to the IGL. A, Locations of NPY-IR cells of the incipient IGL on days E11-E14. $B$, NPY-IR cells scattered along the migration route on E12 (scale bar, $50 \mu \mathrm{m}$ ). $C$, in the IGL on E14 (scale bar, $50 \mu \mathrm{m}$ ); $D$, NPY-IR cell on E12 showing the typical ellipsoid shape, a leading process (small arrow), NPY-IR in the axon hillock (arrowhead) and a trailing process (double arrows). Scale bar, $5 \mu \mathrm{m}$. 

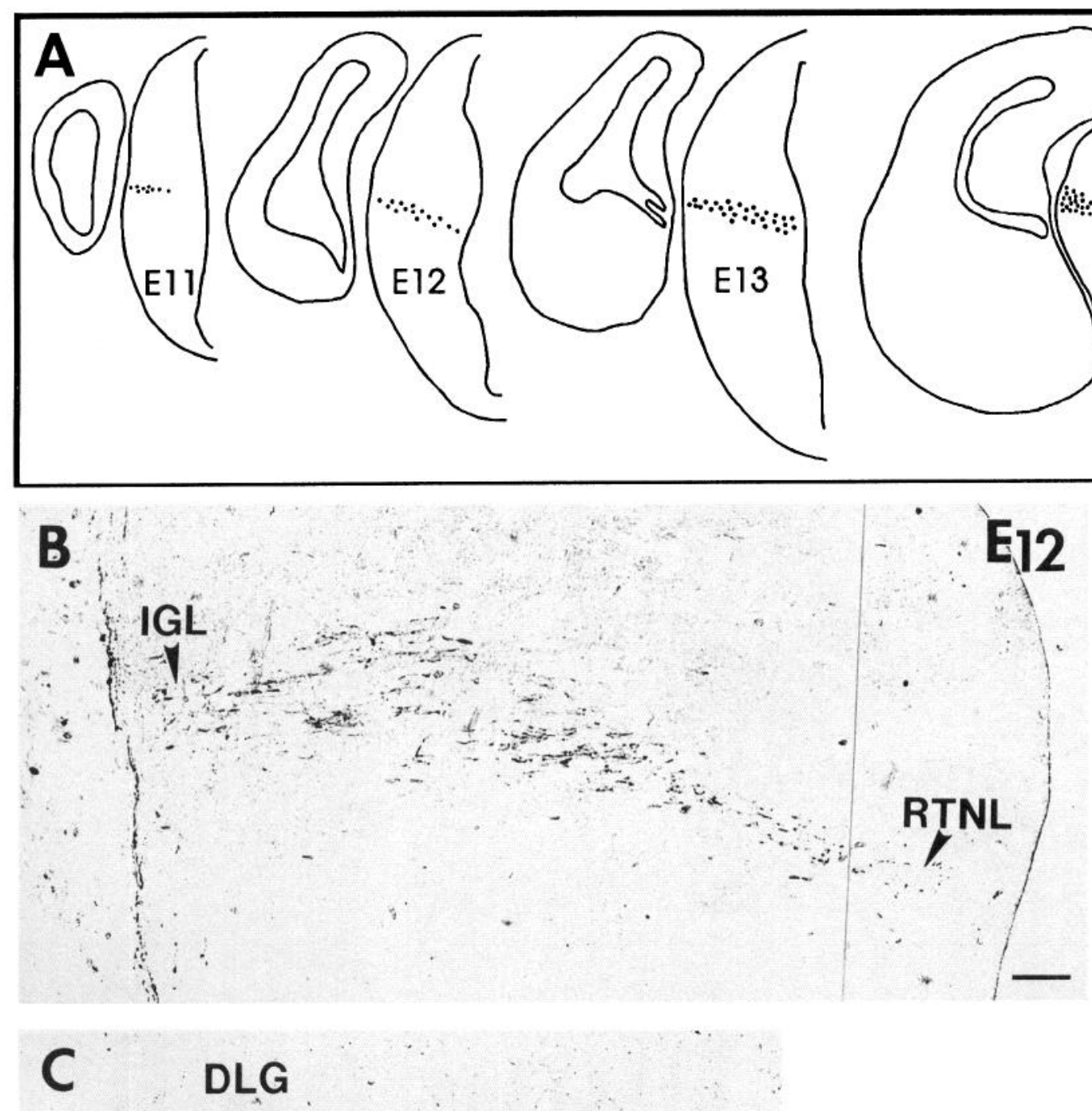

- IGL ,

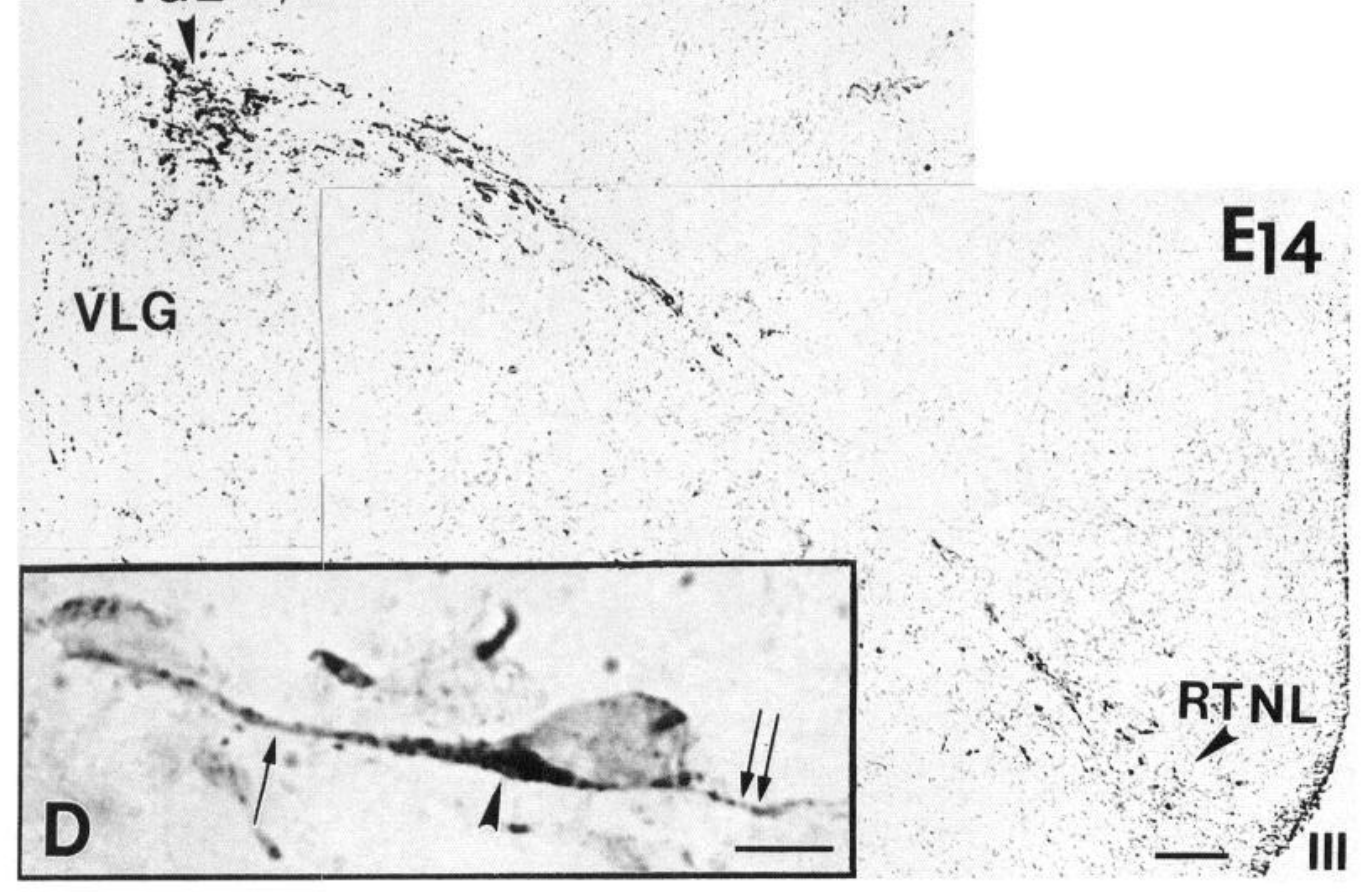



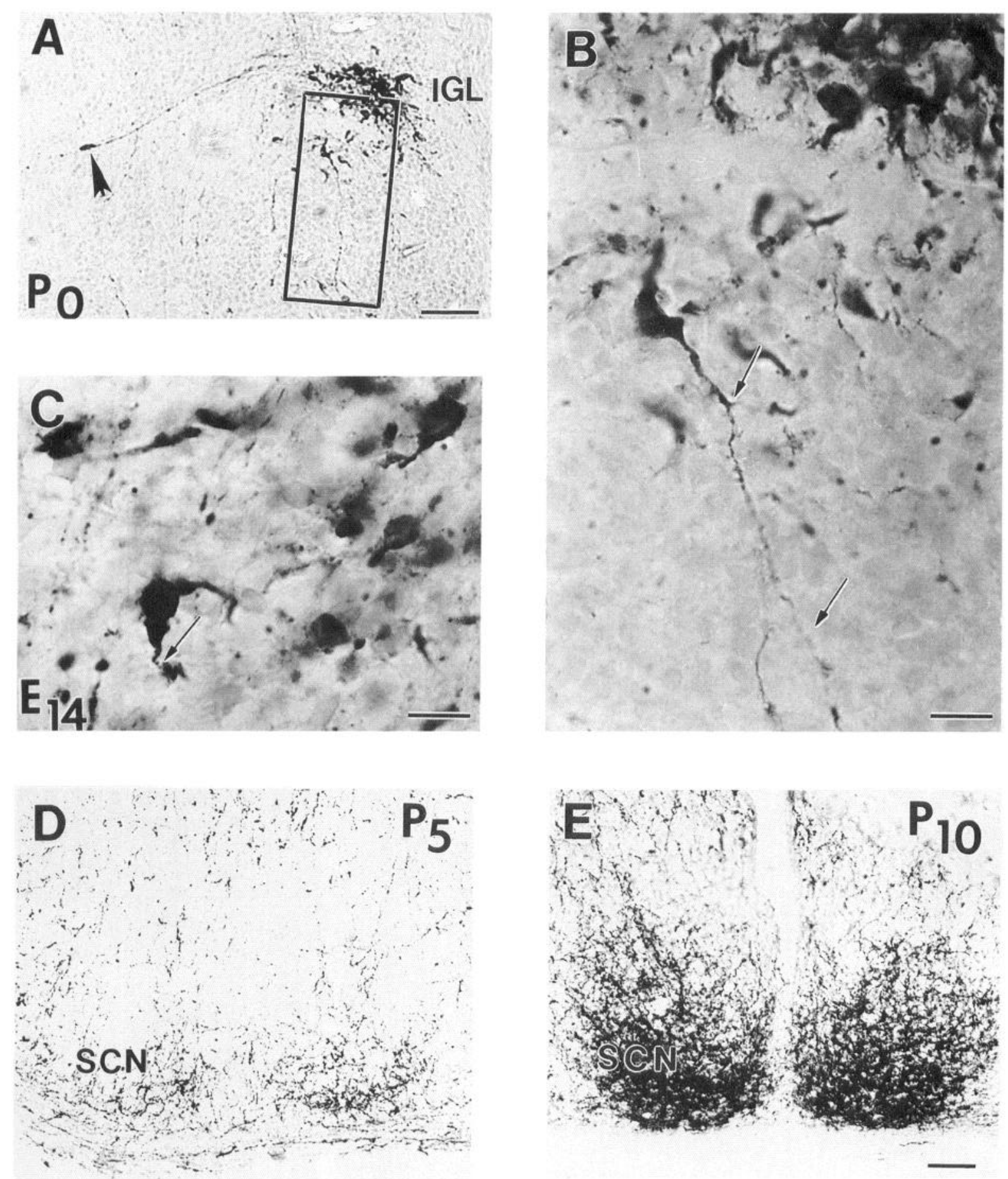

Figure 8. Reorientation of NPY-IR cells in the IGL and formation of the GHT. A, NPY-IR cells in the P0 IGL showing a laggard cell (arrowhead) with a long leading process and cell projecting ventrally into the GHT (in the box). Scale bar, $30 \mu \mathrm{m}$. B, Enlargement of the NPY-IR cell (boxed in $A$ contributing to the GHT. Scale bar, $10 \mu \mathrm{m}$. $C$, Reoriented NPY-IR cell with a short, ventrally extending, axon (arrow). Initial penetration of GHT NPY-IR fibers into the ventral SCN occurs on P3 with more substantial arborization later. Scale bar, 10 $\mu \mathrm{m} . D, \mathrm{P} 5 . E$, P10-adult-like NPYIR plexus. Scale bar, $50 \mu \mathrm{m}$.

IGL (Morin et al., 1992). Papez (1940) considered the VLG to be developmentally related to the reticular nucleus. Altman and Bayer (1979a,b) initially grouped the VLG, zona incerta, subthalamic nucleus, and reticular thalamic nucleus as "ventral thalamus" with similar, early times of origin. Their (Altman and Bayer, 1988) reexamination of reticular thalamic nucleus development revealed an origin substantially different from that of the VLG, but approximately the same as observed here for the hamster IGL. In fact, the most dorsolateral extreme of the embryonic reticular thalamic nucleus identified by Altman and Bayer (1988, their Fig. 2) adjacent to the fimbria is rostral IGL according to our current definitions (Card and Moore, 1989; Morin et al., 1992). These considerations reconcile the fact that the IGL in the present study appears to originate in or near the 
RTNL (Altman and Bayer, 1988) rather than at the much more dorsal sites that give rise to the classically visual geniculate nuclei (Rakic, 1977; Altman and Bayer, 1989).

One general question emphasized by Rakic (1977) concerns whether migratory neurons are "indifferent," acquiring final specificity at their destination or whether they arrive with a determined fate. As shown by the present results, expression of NPY-IR by early postmitotic neurons enables a fairly strong statement concerning point-to-point specificity between cells derived from a dedicated ventricular proliferative sector in the RTNL and the final IGL destination. Rakic (1972) has demonstrated that a function of the radial glial scaffolding is to preserve such a relationship between the site of neuronal origin in the ventricular zone and the postmigratory destination in a more lateral brain nucleus. Neuron fate, described here in terms of peptide content, migratory destination, and future function, is determined at, or shortly after, the time of neuron birth. A similar pattern of lateral migration by neurons of known peptide content from a medial proliferative site in the ventricular mitotic zone has been described in rat hypothalamus (Okamura et al., 1991).

\section{Origin and distribution of astrocytes in the IGL}

The GFAP-IR hypothalamic sulcus tether has now been identified in the fetal hamster, mouse, and cat (Edwards et al., 1990; Silver et al., 1993; present results). Heretofore, the tether has not been associated with particular morphological developments. The present results show that the tether originates in the ventricular ependyma near the reticular thalamic neuroepithelial lobule immediately ventral to the reticular protuberance and terminates in the IGL. The glia of the tether are distinct from the early (E15-P0) astrocyte-like glia scattered along the lateral border of the entire lateral geniculate complex. By P5, these cells are no longer in evidence within the neuropil, but contribute to the glia limitans. In contrast, astrocytes begin to emerge in the P5 IGL, gradually thereafter attaining the adult pattern. This (1) loss of astrocyte-like cells from the geniculate neuropil, (2) coexpression of VIM- and GFAP-IR in cells and fibers of the tether, (3) displacement of GFAP-IR cell bodies from the ventricular ependyma into the route of the glial tether, and (4) subsequent emergence of astrocytes at the former terminus of the tether strongly suggest that a set of specialized radial glia translocate and transform into astrocytes of the IGL.

The adult IGL is definable as a retinorecipient site containing NPY - and ENK-IR neurons that project to the $\mathrm{SCN}$, posterior limitans nucleus, and contralateral IGL (Morin et al., 1992; Morin and Blanchard, 1994; present results). The entire adult IGL, as recognized by that definition, also contains GFAP-IR astrocytes, but there are very few in adjacent DLG or VLG. This is consistent with our previous observation (Morin et al., 1989) and further demonstrates that the IGL is a substantial feature of the lateral geniculate complex, attaining an adult length of about $2 \mathrm{~mm}$.

\section{Development of the geniculohypothalamic tract}

The major axes of migrating NPY-IR cells are oriented mediolaterally toward the IGL. Upon arrival at E14-E15, many reorient by about $90^{\circ}$ and send axons toward the posterior limitans nucleus dorsally or into the GHT ventrally. A distinction must be made between formation of the GHT and presence of NPYIR fibers within it. We have shown that the hamster IGL also sends numerous ENK-IR axons to the SCN (Morin et al., 1992;

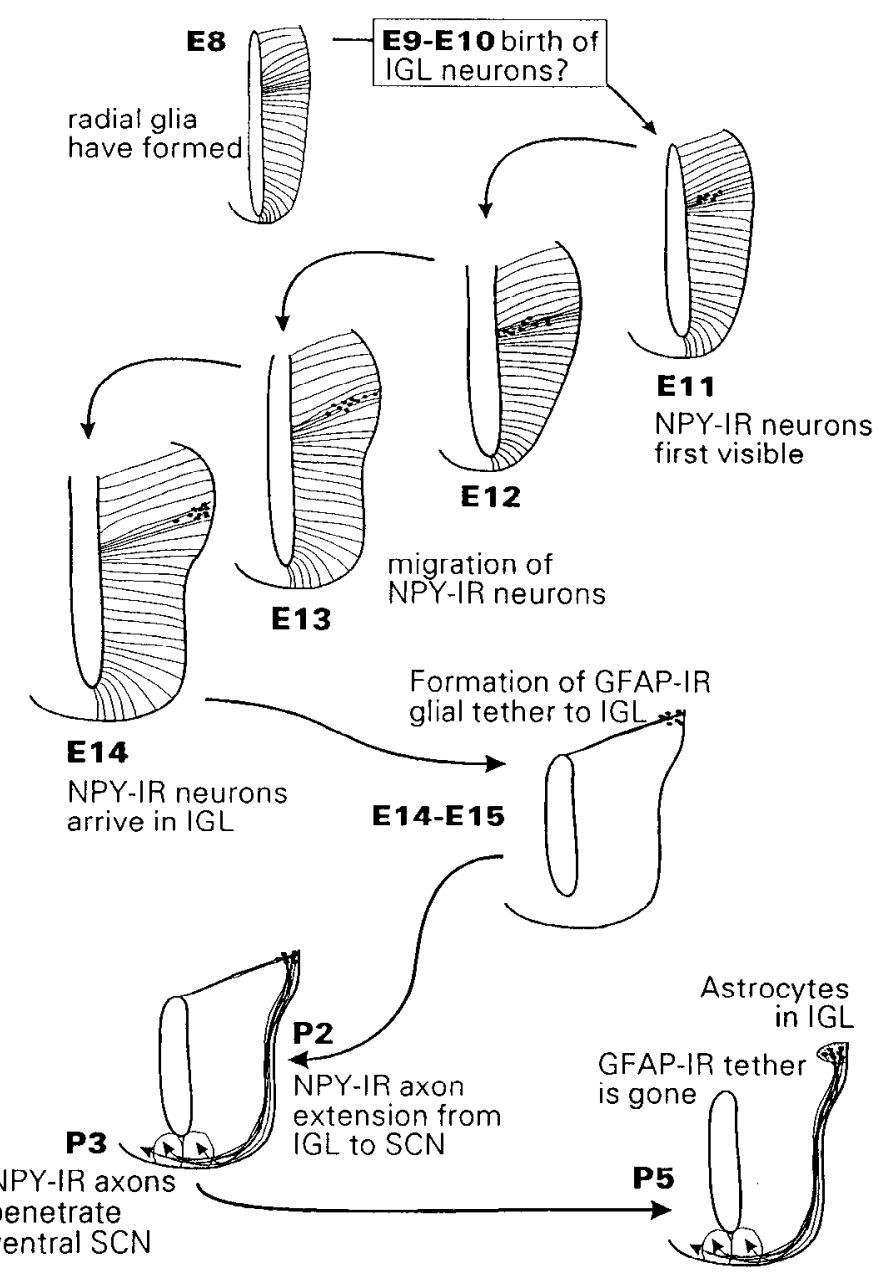

Figure 9. Schematic illustration of proposed IGL development pattern.

Morin and Blanchard, 1994). Neither the origin of ENK-IR cells in the IGL nor the progression of ENK-IR fibers through the GHT to the SCN has been studied. In the rat, many IGL neurons contributing to the GHT are GABAergic, but not NPY- or ENKIR (Card and Moore, 1989; Moore and Speh, 1993). Thus, it is possible that fibers containing neuromodulators other than NPY initiate GHT development and SCN penetration in anticipation of NPY-IR axons to follow.

There has been little study of connections between the IGL and the dorsal thalamus. The present data show NPY-IR fibers exiting the embryonic IGL in a dorsomedial direction. These are presumably directed toward the posterior limitans nucleus (Morin and Blanchard, 1994), although NPY-IR fibers from other cell groups have prevented certainty on this issue. Nevertheless, it is likely that the development of the NPY-IR projection proceeds according to a set of rules similar to that regulating formation of the GHT.

\section{Arrival and arborization of retinal fibers}

The migration of NPY-IR neurons to the IGL and appearance of the glial tether coincides with a major event in hamster visual system development. Rapidly lengthening retinal ganglion cell axons in the hamster optic tract reach the lateral geniculate on E13 (Jhaveri et al., 1991). This "elongation" phase is followed by an approximately $2 \mathrm{~d}$ delay before the "arborization" phase 
of the geniculate nuclei begins on E15.5. At this time, the NPYIR neurons of the IGL also begin to create the GHT by sending fibers ventrally toward the SCN. The relationship between IGI development and retinal innervation of the IGL, VLG, or DLG has not been explicitly studied. However, the IGL may receive retinal fibers later than its neighboring structures (Frost et al., 1979; Frost, 1984; So et al., 1984).

The convergent complexity of neuronal and glial events in the lateral geniculate raises multiple questions concerning the source and nature of putative signals initiating optic fiber ramification. Both neurons and glia have been suggested as regulators of visual system growth patterns (Jhaveri et al., 1991). There is also increasing awareness that anatomical astrocytic "barriers" to axonal penetration organize brain morphology (Steindler, 1993). The entire adult IGL and SCN (Morin et al., 1989; present results) contain dense matrices of astrocytes. The barricr function may be one reason that reinnervation of IGL and $\mathrm{SCN}$, but not of other visual system nuclei, fails following transplantation of embryonic retina to enucleated adults (Radel et al., 1990). The great length of the IGL suggests that in addition to being a nucleus mediating adult circadian rhythmicity, it plays an important functional role in the laminar development of the lateral geniculate complex (Hutchins and Casagrande, 1988, 1990).

\section{Appendix}

\section{Abbreviations}

AMT anteromedian thalamus

CMT centromedian thalamus

$\mathrm{CP} \quad$ cerebral peduncle

DLG dorsolateral geniculate nucleus

fi fimbria

FR fasciculus retroflexus

IGL intergeniculate leaflet

LDVL lateral dorsal thalamus, ventrolateral part

LTN lateral terminal nucleus

MG medial geniculate nucleus

ot optic tract

Rt reticular thalamic nucleus

RTNL reticular neuroepithelial lobule

SCN suprachiasmatic nucleus

st stria terminalis

VLG ventrolateral geniculate nucleus

ZI zona incerta

III third ventricle

\section{References}

Altman J, Bayer SA (1979a) Development of the diencephalon in the rat. IV. Quantitative study of the time of origin of neurons and the internuclear chronological gradients in the thalamus. J Comp Neurol $188: 455-472$

Altman J, Bayer SA (1979b) Development of the diencephalon in the rat. V. Thymidine-radiographic observations on internuclear and intranuclear gradients in the thalamus. J Comp Neurol 188:473-500.

Altman J, Bayer SA (1979c) Development of the diencephalon in the rat. VI. Re-evaluation of the embryonic development of the thalamus on the basis of thymidine-radiographic datings. J Comp Neurol 188: 501-524.

Altman J, Bayer SA (1988) Development of the rat thalamus: III. Time and site of origin and settling pattern of neurons of the reticular nucleus. J Comp Neurol 275:406-428.

Altman J, Bayer SA (1989) Development of the rat thalamus: VI. The posterior lobule of the thalamic neuroepithelium and the time and site of origin and settling pattern of neurons of the lateral geniculate and lateral posterior nuclei. J Comp Neurol 284:581-601.

Botchkina GI, Morin LP (1993) Development of the hamster serotoninergic system: cell groups and diencephalic projections. J Comp Neurol 338:405-431.
Card JP, Moorc RY (1989) Organization of lateral geniculate-hypothalamic connections in the rat. J Comp Neurol 284:135-147.

Choi BH, Lapham LW (1978) Radial glia in the human fetal cerebrum: a combined Golgi, immunofluorescent and electron microscopic study. Brain Res 148:295-311.

Edmondson JC, Hatten ME (1987) Glial-guided granule neuron migration in vitro: a high-resolution time-lapse video microscopic study. J Neurosci 7:1928-1934.

Edwards MA, Yamamoto M, Caviness VS Jr (1990) Organization of radial glia and related cells in the developing murine CNS. An analysis based upon a new monoclonal antibody marker. Neuroscience 36 : 121-144.

Frost DO (1984) Axonal growth and target selection during development: retinal projections to the ventrobasal complex and other "nonvisual" structures in neonatal Syrian hamsters. J Comp Neurol 230:576-592.

Frost DO, So K-F, Schneider GE (1979) Postnatal development of retinal projections in Syrian hamsters: a study using autoradiographic and anterograde degeneration techniques. Neuroscience 4:1649-1677.

Gregory WA, Edmondson JC, Hatten ME, Mason CA (1988) Cytology and neuron-glial apposition of migrating cerebellar granule cells in vitro. J Neurosci 8:1728-1738.

Harrington ME, Rusak B (1989) Photic responses of geniculo-hypothalamic tract neurons in the Syrian hamster. Vis Neurosci 2:367375.

Harrington ME, Nance DM, Rusak B (1987) Double-labeling of neuropeptide $\mathrm{Y}$-immunoreactive neurons which project from the geniculate to the suprachiasmatic nuclei. Brain Res 410:275-282.

Hatten ME (1990) Riding the glial monorail: a common mechanism for glial-guided neuronal migration in different regions of the developing mammalian brain. Trends Neurosci 13:179-184.

Hutchins JB, Casagrande VA (1988) Glial cells develop a laminar pattern before neuronal cells in the lateral geniculate nucleus. Proc Natl Acad Sci USA 85:8316-8320.

Hutchins JB, Casagrande VA (1990) Development of the lateral geniculate nucleus: interactions between retinal afferent, cytoarchitectonic, and glial cell process lamination in ferrets and tree shrews. J Comp Neurol 298:113-128.

Jhaveri S, Edwards MA, Schneider GE (1991) Initial stages of retinofugal axon development in the hamster: evidence for two distinct modes of growth. Exp Brain Res 87:371-382.

Johnson RF, Smale L, Moore RY, Morin LP (1988) Lateral geniculate lesions block circadian phase shift responses to a benzodiazepine. Proc Natl Acad Sci USA 85:5301-5304.

Lavialle M, Servière J (1993) Circadian fluctuations in GFAP distribution in the Syrian hamster suprachiasmatic nucleus. Neuroreport 4:1243-1246.

Levitt P, Rakic P (1980) Immunoperoxidase localization of glial fibrillary acidic protein in radial glial cells and astrocytes of the developing rhesus monkey brain. J Comp Neurol 193:815-840.

Moore RY (1989) The geniculohypothalamic tract in monkey and man. Brain Res 486:190-194.

Moore RY, Speh JC (1993) GABA is the principal neurotransmitter of the circadian system. Neurosci Lett 150:112-116.

Morin LP (1994) The circadian visual system. Brain Res Rev 67:102127.

Morin LP, Blanchard J (in press) Organization of the hamster intergeniculate leaflet: NPY and ENK projections to the suprachiasmatic nucleus, intergeniculate leaflet and posterior limitans nucleus. Vis Neurosci, in press.

Morin LP, Johnson RF, Moore RY (1989) Two brain nuclei regulating circadian rhythms are identified by GFAP immunoreactivity in hamsters and rats. Neurosci Lett 99:55-60.

Morin LP, Blanchard JH, Moore RY (1992) Intergeniculate leaflet and suprachiasmatic nucleus organization and connections in the hamster. Vis Neurosci 8:219-230.

Okamura Y, Kawano H, Daikoku S (1991) Spatial-temporal appearance of developing immunoreactive TRH neurons in the neuroepithelial wall of the diencephalon. Dev Brain Res 63:21-31.

Papez JW (1940) The embryologic development of the hypothalamic area in mammals. Res Publ Assoc Nerv Ment Dis 20:31-51.

Pixley SKR, de Vellis J (1984) Transition between immature radial glia and mature astrocytes studied with a monoclonal antibody to vimentin. Dev Brain Res 15:201-209.

Radel JD, Hankin MH, Lund RD (1990) Proximity as a factor in the 
innervation of host brain regions by retinal transplants. J Comp Neurol 300:211-229.

Rakic P (1971) Neuron-glia relationship during granule cell migration in developing cerebellar cortex. J Comp Neurol 141:283-312.

Rakic P (1972) Mode of cell migration to the superficial layers of fetal monkey neocortex. J Comp Neurol 145:61-84.

Rakic P (1977) Genesis of the dorsal lateral geniculate nucleus in the rhesus monkey: site and time of origin, kinetics of proliferation, routes of migration and pattern of distribution neurons. J Comp Neurol 176: 23-62.

Rakic P (1988) Specification of cerebral cortical areas. Science 241: 170-176.

Rhoades RW, Hsu L, Parfett G (1979) An electronmicroscopic analysis of the optic nerve in the golden hamster. J Comp Neurol 186: 491-504.

Rhoades RW, Mooney RD, Chiaia NL, Bennett-Clarke CA (1990) Development and plasticity of the serotoninergic projection to the hamster's superior colliculus. J Comp Neurol 299:151-166.

Schmechel DE, Kakic P (1979) A Golgi study of radial glial cells in developing monkey telencephalon: morphogenesis and transformation into astrocytes. Anat Embryol (Berl) 156:115-152.
Silver J, Edwards MA, Levitt P (1993) Immunocytochemical demonstration of early appearing astroglial structures that form boundaries and pathways along axon tracts in the fetal brain. J Comp Neurol 328:415-436.

So K-F, Schneider GE, Frost DO (1978) Pustnatal development of retinal projections to the lateral geniculate body in Syrian hamsters. Brain Res 142:343-352.

So K-F, Woo HH, Jen LS (1984) The normal and abnormal postnatal development of retinogeniculate projections in golden hamsters: an anterograde horseradish peroxidase tracing study. Dev Brain Res 12: 191-205.

Steindler DA (1993) Glial boundaries in the developing nervous system. Annu Rev Neurosci 16:445-470.

Voigt T (1989) Development of glial cells in the cerebral wall of ferrets: direct tracing of their transformation from radial glia into astrocytes. J Comp Neurol 289:74-88.

Zhang DX, Rusak B (1989) Photic sensitivity of geniculate neurons that project to the suprachiasmatic nuclei or the contralateral geniculate. Brain Res 504:161-164. 